\title{
DETERMINAÇÃO RÁPIDA DE OSELTAMIVIR EM CÁPSULAS POR CROMATOGRAFIA LÍQUIDA DE ULTRAEFICIẾNCIA COM DETECTOR POR ARRANJO DE DIODOS
}

\author{
Marina Venzon Antunes, Bárbara Estefânia Fraga Griebeler e Rafael Linden* \\ Universidade Feevale, Rod. RS 239, n. 2755, 93352-000 Novo Hamburgo - RS, Brasil
}

Recebido em 25/10/10; aceito em 1/2/11; publicado na web em 29/3/11

\begin{abstract}
RAPID DETERMINATION OF OSELTAMIVIR IN CAPSULES BY ULTRA PERFORMANCE LIQUID CHROMATOGRAPHY WITH PHOTODIODE ARRAY DETECTOR. A simple and rapid ultra-performance liquid chromatographic method for determination of oseltamivir in capsules was developed and validated. The mobile phase consisted of $5 \mathrm{mmol} / \mathrm{L}$ triethylammonium buffer (pH 3.0) and acetonitrile (70:30, v/v). Separation was performed in a Hypersil Gold ${ }^{\circledast}$ column, with octylsilil as stationary phase $(100$ x 2.1 $\mathrm{mm}$, p.d. $1.9 \mu \mathrm{m}$ ). Chromatography run time was $1.2 \mathrm{~min}$. The method presented adequate specificity, linearity, precision, ruggedness and accuracy and was adequate for determination of oseltamivir in capsules.
\end{abstract}

Keywords: oseltamivir; ultra performance liquid chromatography; photodiode array detector.

\section{INTRODUÇÃO}

O Oseltamivir (OSV) é o primeiro inibidor oral seletivo da neuroaminidase disponível para a profilaxia e o tratamento da influenza A e B, sendo um pró-fármaco rapidamente hidrolisado via esterases hepáticas a seu metabólito ativo, o carboxilato de oseltamivir. ${ }^{1}$ Sua fórmula estrutural é apresentada na Figura 1.<smiles>CCOC(=O)C1=C[C@H](OC(CC)CC)[C@H](NC(C)=O)[C@H](N)C1</smiles>

Figura 1. Fórmula estrutural do Oseltamivir

O OSV foi desenvolvido pela Gilead Sciences e inicialmente comercializado pela Hoffmann La Roche sob o nome comercial de Tamiflu , disponível na forma de fosfato de oseltamivir em cápsulas com 30, 45 ou $75 \mathrm{mg}$ ou suspensão oral com $12 \mathrm{mg} / \mathrm{mL} .{ }^{2,3}$ Segundo a Organização Mundial da Saúde (OMS), a dose recomendada para o tratamento de adultos é de $75 \mathrm{mg}$ duas vezes ao dia, por um período de 5 dias, e, para a profilaxia é de $75 \mathrm{mg}$ uma vez ao dia, por pelo menos 7 dias. ${ }^{4}$

No ano de 2009, durante o surto mundial de gripe causado pelo vírus influenza A H1N1, foram oficialmente notificados mais de 414.945 casos de pessoas infectadas, dentre os quais pelo menos 5.000 foram a óbito até o dia 27/9/2009. ${ }^{5}$ Em decorrência do aumento da demanda mundial por este medicamento, atualmente considerado o principal agente antiviral disponível para combater epidemias graves ou surto de pandemia de gripe, outras empresas farmacêuticas em diferentes países estão produzindo OSV sob licença da Roche ${ }^{\circledR} .{ }^{1,2}$ Além disso, existem ainda diversos relatos da comercialização irregular de

*e-mail: rafael.linden@feevale.br produtos similares de baixa qualidade, com quantidades alteradas do princípio ativo e, até mesmo, de produtos falsificados. ${ }^{6}$

Sabe-se que o tratamento com medicamentos de qualidade inadequada pode levar à falha terapêutica, causando sérios danos à saúde do paciente. ${ }^{7}$ Desta forma, é importante a disponibilidade de metodologias adequadas para a caracterização da qualidade dos produtos farmacêuticos, através da sua identificação e quantificação.

Alguns métodos para a quantificação de OSV em preparações farmacêuticas já foram descritos na literatura. Nestes trabalhos, as determinações analíticas foram realizadas através de colorimetria, ${ }^{8}$ fluorimetria, ${ }^{9}$ eletroforese capilar, ${ }^{3}$ ou por cromatografia líquida de alta eficiência com detecção por ultravioleta, ${ }^{1,2,8,10-12}$ fluorescência ${ }^{13,14}$ ou espectrometria de massas. ${ }^{15-17}$

A cromatografia líquida de ultraeficiência (CLUE) é um dos avanços mais recentes das técnicas de separação, baseando-se nos mesmos princípios da cromatografia líquida de alta eficiência (CLAE). Porém, esta técnica utiliza fases estacionárias com partículas menores, o que possibilita análises mais rápidas, menor consumo de solventes e detectabilidade aproximadamente 3 vezes maior, se comparada à CLAE. ${ }^{18}$ Não foram encontradas, até o presente momento, na literatura referências sobre a utilização da CLUE para a avaliação da qualidade de formas farmacêuticas contendo OSV. Desta forma, neste trabalho apresentamos um método simples e rápido, empregando CLUE para a determinação de Oseltamivir em cápsulas, como ferramenta para a caracterização de produtos supostamente contendo esta substância e comercializados de forma irregular no Brasil.

\section{PARTE EXPERIMENTAL}

\section{Materiais}

Padrão de trabalho

O padrão analítico de Fosfato de Oseltamivir foi doado pela Hoffmann La Roche AG (Basel, Suíça).

\section{Reagentes}

Metanol foi adquirido da F. Maia (Cotia, Brasil). Acetonitrila e ácido fosfórico 85\% foram obtidos da Merck (Darmstadt, Alemanha). 
A solução tampão fosfato de trietilamônio $1 \mathrm{M}$ foi obtida da Fluka (Buchs, Suíça). Os excipientes polivinilpirrolidona, celulose e talco foram fornecidos pelo Laboratório de Desenvolvimento Galênico da Universidade Federal do Rio Grande do Sul (Porto Alegre, Brasil). Água purificada foi obtida através de um sistema Elga Purelab Ultra ${ }^{\circledR}$ da Elga Labwater (Lane End, Reino Unido).

\section{Amostras}

Foram analisadas 8 especialidades farmacêuticas contendo OSV em cápsulas, sendo uma formulação de referência, disponível no mercado farmacêutico brasileiro, e as demais similares produzidas em outros países da América Latina, eventualmente comercializados no Brasil em estabelecimentos não farmacêuticos e através da internet.

\section{Preparação da fase móvel}

A fase móvel foi composta de tampão trietilamônio $5 \mathrm{mmol} / \mathrm{L}$ ( $\mathrm{pH}$ $3,0)$ e acetonitrila (70:30, v/v). O tampão trietilamônio $5 \mathrm{mmol} / \mathrm{L}$ foi preparado através da diluição da solução mãe 1 mol/L em água ultrapurificada e posteriormente filtrado através de membrana de acetato de celulose com poros de 0,2 $\mu \mathrm{m}$ (Waters, EUA). Antes da utilização, a fase móvel foi desgaseificada em banho ultrassônico por $5 \mathrm{~min}$.

\section{Condições cromatográficas}

A análise foi realizada empregando um sistema de cromatografia líquida de ultraeficiência Acquity ${ }^{\circledR}$ com detector por arranjo de diodos (CLUE-DAD). O sistema foi gerenciado pelo programa Empower ${ }^{\circledR}$ e composto por bomba binária, autoamostrador, forno de coluna e detecção por arranjo de fotodiodos, todos provenientes da Waters (Milford, EUA). A separação foi realizada em uma coluna Hypersil Gold $^{\circledR}$ (Thermo, EUA), contendo octilsílica como fase estacionária com dimensões de 100 x 2,1 mm e diâmetro da partícula de 1,9 $\mu \mathrm{m}$. A coluna foi mantida a $50{ }^{\circ} \mathrm{C}$ durante a análise, com vazão da fase móvel de 0,5 mL/min. Os cromatogramas foram monitorados em 220 nm, com aquisição de espectros de varredura entre 200 e $380 \mathrm{~nm}$. A corrida cromatográfica teve duração de 1,2 min.

\section{Método}

\section{Preparo da solução padrão de Oseltamivir}

Para o preparo da solução mãe de $1 \mathrm{mg} / \mathrm{mL}$ de Oseltamivir como base livre, foram transferidos $13,137 \mathrm{mg}$ de fosfato de Oseltamivir em balão volumétrico com capacidade de $10,00 \mathrm{~mL}$ e o volume foi completado com metanol até o traço de aferição.

\section{Preparo das amostras}

Todo o conteúdo da cápsula (75 mg de oseltamivir, base livre e excipientes) foi transferido para balão volumétrico de $100,00 \mathrm{~mL}$ ao qual foram acrescentados $90 \mathrm{~mL}$ de água ultrapurificada. O balão foi submetido a banho ultrassônico por $10 \mathrm{~min}$ e, em seguida, o volume foi completado com água (solução com concentração teórica de oseltamivir de $750 \mu \mathrm{g} / \mathrm{mL}$ ). A solução foi transferida para tubo de ensaio e centrifugada a 3.000 g por $10 \mathrm{~min}$. Uma alíquota de $60 \mu \mathrm{L}$ do sobrenadante foi diluída até $1000 \mu \mathrm{L}$ com fase móvel, resultando em uma solução com concentração teórica de $45 \mu \mathrm{g} / \mathrm{mL}$. Desta solução foram injetados $2 \mu \mathrm{L}$ no sistema CLUE-DAD. Todas as amostras foram analisadas em triplicata.

\section{Validação}

O método desenvolvido foi validado de acordo com os parâmetros determinados pelo "Guia para validação de métodos analíticos e bioanalíticos", conforme resolução n 899 da Agência Nacional de Vigilância Sanitária (ANVISA). Foram avaliados os parâmetros especificidade, linearidade, precisão intra e interdias, exatidão e robustez. ${ }^{19}$

\section{Especificidade}

Considerou-se o método específico caso não houvesse a presença de picos interferentes no mesmo tempo de retenção do fosfato de oseltamivir nas análises dos excipientes. Além disto, avaliou-se a pureza dos picos cromatográficos pelos espectros de absorção gerados pela detecção por arranjo de diodos para o OSV. Preparou-se a mistura física de cada um dos seguintes adjuvantes farmacêuticos: celulose 43,5 mg; polivinilpirrolidona 4,35 mg e talco 1,45 mg, os quais, segundo informações da bula do medicamento Tamiflu , integram a composição das cápsulas desta especialidade farmacêutica. Esta mistura foi transferida para um balão volumétrico com capacidade de 100,00 mL, ao qual foram adicionados $90,00 \mathrm{~mL}$ de água ultrapurificada. A seguir, adotou-se processamento idêntico ao descrito no subitem "Preparo das amostras".

\section{Linearidade e intervalo}

Para a construção da curva de calibração, foi utilizada a média de 3 determinações de cada calibrador em 5 níveis diferentes. A curva de calibração foI construída através do estabelecimento da correlação entre a área do pico de OSV (y) e as concentrações adicionadas (x). A curva foi avaliada com base no coeficiente de determinação $\left(\mathrm{r}^{2}\right)$. Foram utilizados calibradores nas concentrações de 10; 25; 50; 75 e $100 \mu \mathrm{g} / \mathrm{mL}$ de OSV, preparados a partir da diluição da solução padrão $1 \mathrm{mg} / \mathrm{mL}$ em fase móvel.

\section{Precisão e exatidão}

A precisão e a exatidão do método foram avaliadas através das análises em três níveis de concentração, realizadas em triplicata e repetidas em 3 dias diferentes. As soluções foram preparadas em solvente contendo os excipientes nas concentrações indicadas no subitem "Especificidade". As medidas obtidas para cada nível de concentração foram submetidas à análise de variância (ANOVA - fator único) para estimar a precisão intraensaios e a precisão interensaios, ambas expressas como coeficiente de variação porcentual (C.V.\%).A exatidão foi determinada a partir dos mesmos resultados empregados no cálculo da precisão e expressa como percentagem média obtida do valor teórico de cada calibrador. As concentrações utilizadas no estudo de precisão e exatidão foram 20,40 e $80 \mu \mathrm{g} / \mathrm{mL}$, preparadas a partir da diluição da solução padrão de OSV $1 \mathrm{mg} / \mathrm{mL}$ em solução contendo os excipientes.

\section{Robustez}

Foi avaliada a suscetibilidade do método a variações nas condições analíticas empregadas. Foram analisadas as variações de $\mathrm{pH}(2,7$; $3,0$ e 3,3$)$, temperatura $\left(49,50\right.$ e $\left.51^{\circ} \mathrm{C}\right)$ e vazão da fase móvel $(0,45$; 0,50 e $0,55 \mathrm{~mL} / \mathrm{min}$ ), através da análise em triplicata da solução de OSV a $20 \mu \mathrm{g} / \mathrm{mL}$. Os resultados das determinações foram submetidos à análise de variância (ANOVA - fator único).

\section{RESULTADOS E DISCUSSÃO}

Nas condições analíticas utilizadas, o tempo de análise cromatográfica foi consideravelmente curto, de apenas 1,2 min, com o tempo de retenção do Oseltamivir ficando em 0,95 min (Figura 2). A vazão da fase móvel de $0,5 \mathrm{~mL} / \mathrm{min}$, em uma análise cromatográfica de 1,2 min, resultou em consumo de apenas $0,6 \mathrm{~mL}$ de fase móvel durante a análise. O emprego da vazão da fase móvel reduzida, aliado ao curto tempo da análise cromatográfica, permite economia no consumo de solventes e ganho de tempo nas análises, sendo estas as principais 
vantagens do emprego da CLUE para a análise deste fármaco. Comparado aos métodos descritos na literatura com emprego da CLAE ocorreu redução de 4 vezes, pelo menos, no tempo de análise cromatográfica. ${ }^{1,2,8,10-12}$
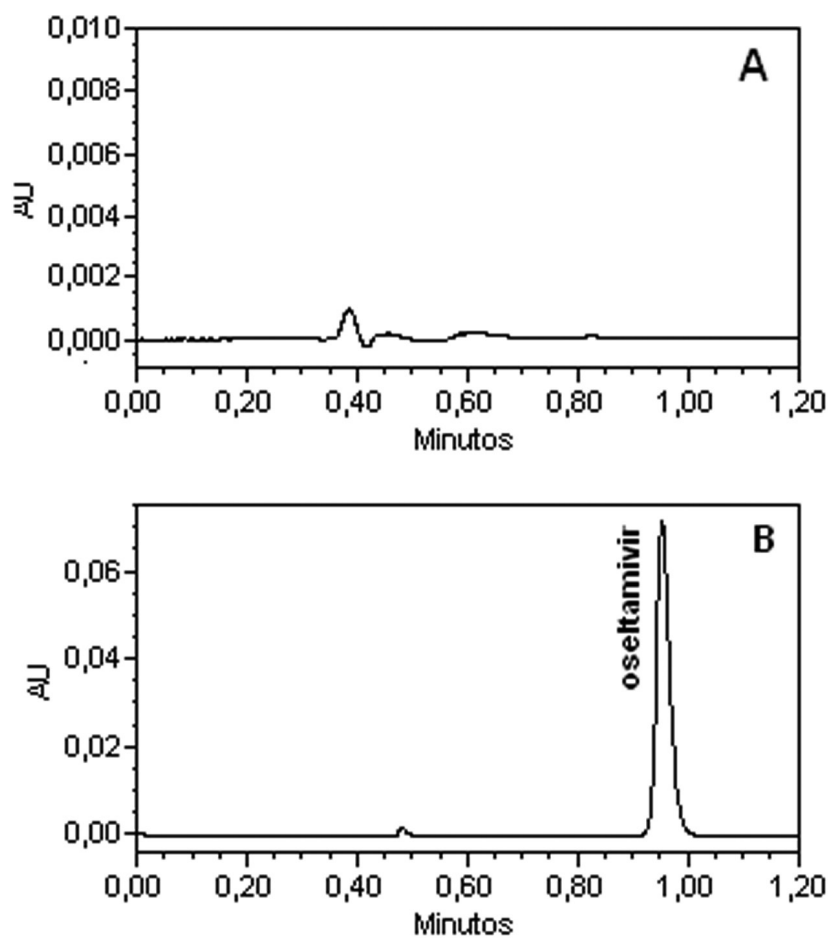

Figura 2. Cromatograma monitorado em $220 \mathrm{~nm}$. A. Excipientes: celulose 26,1 $\mu \mathrm{g} / \mathrm{mL}$; polivinilpirrolidona $2,6 \mu \mathrm{g} / \mathrm{mL}$ e talco $0,9 \mu \mathrm{g} / \mathrm{mL}$; B. solução Oseltamivir $50,0 \mu \mathrm{g} / \mathrm{mL}$

No presente trabalho o tempo despendido na preparação das amostras foi de aproximadamente $40 \mathrm{~min}$, considerando-se os tempos de incubação no ultrassom e centrifugação, de 10 min cada. No trabalho apresentado por Lindegardh et al. ${ }^{10}$ para a determinação de OSV em especialidades farmacêuticas, empregando CLAE com detecção por UV, o tempo para a preparação das amostras (de cerca de $60 \mathrm{~min}$ ) e de corrida cromatográfica (de $7 \mathrm{~min}$ ) foram superiores aos propostos por este trabalho. ${ }^{10}$

Nos métodos desenvolvidos por Narasimhan et al. ${ }^{2}$ e JosephCharles et al., ${ }^{11}$ que também utilizaram CLAE-UV para avaliar cápsulas de similares de OSV, apesar do preparo simples e rápido das amostras, as corridas cromatográficas foram de 12 e $5 \mathrm{~min}$, com vazão da fase móvel de 1 e 1,2 $\mathrm{mL} / \mathrm{min}$, respectivamente. Por fim, Green et al. ${ }^{8}$ utilizaram colorimetria seguida por CLAE para avaliação das cápsulas de produtos genéricos de OSV, sendo que a CLAE teve tempo de análise cromatográfica de $7 \mathrm{~min}$, com vazão de fase móvel de $1 \mathrm{~mL} / \mathrm{min}$. Nos trabalhos acima citados, as separações cromatográficas foram realizadas com a fase móvel mantida em vazão de 1 a $1,2 \mathrm{~mL} / \mathrm{min}$, consumindo de 6 a $12 \mathrm{~mL}$ de solvente por análise, volumes que são pelo menos 10 vezes maiores do que os empregados neste trabalho.

$\mathrm{Na}$ avaliação da especificidade do método aqui apresentado, não foi observada a presença de picos interferentes na análise da mistura física dos excipientes, não apresentando assim tempos de retenção semelhantes ao OSV. Além disto, através da detecção de arranjo de diodos, foi possível a aquisição do espectro de absorbância do oseltamivir na região do ultravioleta e a verificação da pureza espectral das amostras. A conjugação destes fatos permite considerar o método específico, fornecendo alto grau de confiabilidade na identificação do fármaco (Figura 2).

O método se apresentou linear na faixa de concentrações de 10 a $100 \mu \mathrm{g} / \mathrm{mL}$, uma vez que o coeficiente de determinação $\left(\mathrm{r}^{2}\right)$ foi de 0,9996, com intercepto 149,04 e inclinação 6514,6 . Os parâmetros precisão intraensaios e precisão interensaios estiveram entre 1,47 a $2,68 \%$, demonstrando a elevada precisão do método, especialmente devido ao preparo simplificado das amostras. A exatidão determinada foi de 96 a 99\%, também dentro dos critérios de aceitação preconizados pela ANVISA e normativas internacionais para tais métodos analíticos ${ }^{19,20}$ (Tabela 1$)$.

Tabela 1. Resultados dos ensaios de precisão e exatidão

\begin{tabular}{cccc}
\hline $\begin{array}{c}\text { Oseltamivir } \\
(\mu \mathrm{g} / \mathrm{mL})\end{array}$ & $\begin{array}{c}\text { Precisão intradia } \\
(\text { C.V. } \%)\end{array}$ & $\begin{array}{c}\text { Precisão interdia } \\
(\text { C.V. } \%)\end{array}$ & $\begin{array}{c}\text { Exatidão } \\
(\%)\end{array}$ \\
\hline 20 & 5,78 & 2,68 & 96 \\
40 & 3,08 & 2,15 & 98 \\
80 & 1,47 & 2,08 & 99 \\
\hline
\end{tabular}

O método pode ser considerado robusto, visto que os resultados das áreas encontrados nas determinações da solução de oseltamivir $20 \mu \mathrm{g} / \mathrm{mL}$ não apresentaram diferença estatisticamente significativa quando foram variadas as condições de $\mathrm{pH}$, vazão da fase móvel e temperatura no forno da coluna $(\mathrm{P}=0,594)$ (Tabela 2$)$.

A partir do método validado, foram realizadas análises de 8 cápsulas de especialidades farmacêuticas contendo oseltamivir. Os resultados encontrados (Tabela 3) estão dentro da faixa aceitável estabelecida pela ANVISA (90,0 a 110,0\%), ${ }^{21}$ com dosagens de oseltamivir na faixa de 100,4 a 108,5\%. Estes resultados estão em concordância com os artigos publicados anteriormente, nos quais os produtos avaliados também apresentaram resultados dentro da faixa aceitável.

O método desenvolvido permite a realização de análises de um grande número de amostras em um curto período de tempo.

Tabela 2. Resultados do ensaio de robustez

\begin{tabular}{|c|c|c|c|c|c|c|}
\hline \multicolumn{3}{|c|}{ Condição analítica } & \multirow{2}{*}{ Réplica $1(\mu \mathrm{g} / \mathrm{mL})$} & \multirow{2}{*}{ Réplica $2(\mu \mathrm{g} / \mathrm{mL})$} & \multirow{2}{*}{ Réplica $3(\mu \mathrm{g} / \mathrm{mL})$} & \multirow{2}{*}{ valor-P } \\
\hline $\mathrm{pH}$ & $\mathrm{T}\left({ }^{\circ} \mathrm{C}\right)$ & $\mathrm{V}(\mathrm{mL} / \mathrm{min})$ & & & & \\
\hline 3,0 & 49 & 0,50 & 20,17 & 20,16 & 20,19 & 0,594 \\
\hline 3,0 & 50 & 0,50 & 20,14 & 20,27 & 20,12 & \\
\hline 3,0 & 50 & 0,45 & 20,02 & 20,13 & 20,27 & \\
\hline 3,0 & 50 & 0,55 & 19,99 & 20,12 & 20,07 & \\
\hline 3,0 & 51 & 0,50 & 20,03 & 19,99 & 20,22 & \\
\hline 3,3 & 50 & 0,50 & 20,38 & 20,10 & 20,09 & \\
\hline 2,7 & 50 & 0,50 & 20,04 & 20,05 & 20,17 & \\
\hline
\end{tabular}

T: temperatura; V: vazão fase móvel 
Tabela 3. Resultados das determinações de Oseltamivir em especialidades farmacêuticas

\begin{tabular}{lccc}
\hline $\begin{array}{l}\text { Especialidade } \\
\text { farmacêutica }\end{array}$ & $\begin{array}{c}\text { Concentração } \\
\text { determinada* }(\mu \mathrm{g} / \mathrm{mL})\end{array}$ & $\begin{array}{c}\text { OSV } \\
(\mathrm{mg} / \text { capsula })\end{array}$ & $\begin{array}{c}\text { Teor } \\
(\%)\end{array}$ \\
\hline Referência & 45,2 & 75,4 & 100,5 \\
Similar 1 & 45,1 & 75,3 & 100,4 \\
Similar 2 & 46,2 & 76,9 & 102,6 \\
Similar 3 & 47,1 & 78,6 & 104,8 \\
Similar 4 & 48,8 & 81,4 & 108,5 \\
Similar 5 & 45,2 & 75,3 & 100,4 \\
Similar 6 & 46,2 & 77,0 & 102,7 \\
Similar 7 & 45,5 & 75,8 & 101,1 \\
\hline
\end{tabular}

$*_{\mathrm{n}}=3$

Assim, podemos constatar que o emprego da cromatografia líquida de ultraeficiência é eficiente para a determinação de oseltamivir em especialidades farmacêuticas.

\section{CONCLUSÃO}

Foi desenvolvido um método simples e rápido para determinação de oseltamivir em cápsulas empregando cromatografia líquida de ultraeficiência. O tempo total de análise cromatográfica foi de 1,2 min, com consumo de fase móvel de apenas 0,6 mL. O método foi aplicado a 8 especialidades farmacêuticas, que apresentaram teores de oseltamivir compatíveis com os requerimentos da ANVISA. O presente método é uma ferramenta útil para a caracterização de cápsulas supostamente contendo Oseltamivir.

\section{REFERÊNCIAS}

1. Bahrami, G.; Mohammadia, B.; Kiani, A.; J. Chromatogr., B: Anal. Technol. Biomed. Life Sci. 2008, 864, 38.

2. Narasimhan, B.; Abida, K.; Srinivas, K.; Chem. Pharm. Bull. 2008, 56, 413.
3. Laborde-Kummer, E.; Gaudin, K.; Joseph-Carles, J.; Gheyouche, R.; Boudis, H.; Dubost, J. P.; J. Pharm. Biomed. Anal. 2009, 50, 544.

4. http://www.who.int/csr/disease/avian_influenza/guidelines/ avianinfluenza_oseltamivir/en/, acessada em Março 2011.

5. http://www.who.int/csr/don/2009_09_18/en/, acessada em Agosto 2010.

6. http://www.crfgo.org.br/site/index.php?page=mostra_noticia\&id=2125, acessada em Março 2011.

7. http://portal.anvisa.gov.br/wps/portal/anvisa/home/medicamentos, acessada em Março 2011.

8. Green, M. D.; Nettey, H.; Wirtz, R. A.; Emerg. Infect. Dis. 2008, 14, 4.

9. Aydogmus, Z.; J. Fluoresc. 2009, 19, 673.

10. Lindegardh, N.; Hien, T. T.; Farrar, J.; Singhasivanon, P.; White, N. J.; Day, N. P. J.; J. Pharm. Biomed. Anal. 2006, 42, 430.

11. Joseph-Charles, J. J.; Geneste, C.; Laborder, K. E.; Gheyouche, R.; Boudis, H.; Dubost, J. P.; J. Pharm. Biomed. Anal. 2007, 44, 1008.

12. Fuke, C.; Ihama, Y.; Miyazaki, T.; Leg. Med. 2008, 10, 83.

13. Eisenberg, E. J.; Cundy, K. C.; J. Chromatogr., B: Anal. Technol. Biomed. Life Sci. 1998, 716, 267.

14. Accinelli, C.; Caracciolo, A. B.; Grenni, P.; Int. J. Environ. Anal. Chem. 2007, 87, 579 .

15. Wiltshire, H.; Wiltshire, B.; Citron, A.; Clarke, T.; Serpe, C.; Gray, D.; Herron, W.; J. Chromatogr., B: Anal. Technol. Biomed. Life Sci. 2000, 745,373 .

16. Lindegardh, N.; Hanpithakpong, W.; Wattanagoon, Y.; Singhasivanon, P.; White, N. J.; Day, N. P. J.; J. Chromatogr., B: Anal. Technol. Biomed. Life Sci. 2007, 859, 74.

17. Heinig, K.; Bucheli, F.; J. Chromatogr., B: Anal. Technol. Biomed. Life Sci. 2008, 876, 129.

18. Maldaner, L.; Jardim, I. C. S. F.; Quim. Nova 2009, 32, 214.

19. http://www.anvisa.gov.br/legis/resol/2003/re/899_03re.htm, acessada em Março 2011.

20. Shah, V. P.; Midha, K. K.; Findlay, J. W.; Hill, H. M.; Hulse, J. D.; McGilveray, I. J.; McKay, G.; Miller, K. J.; Patnaik, R. N.; Powell, M. L.; Tonelli, A; Viswanathan, C. T.; Yacobi, A.; Pharm. Res. 2000, 17, 1551.

21. http://www.anvisa.gov.br/legis/resol/2002/475_02re.htm, acessada em Março 2011. 